$r$ (positiv, wenn $a^{>}>\beta$ ), und es ist $p=\frac{\mathcal{F}}{\mathcal{F}^{\prime}+r}$; ferner lautet die Ablesung

$$
\begin{aligned}
& \text { bez. } A:\left\{\begin{array}{l}
A+\alpha p \\
B+(\mathscr{A}-\beta)
\end{array}\right\} \\
& \text { bez. } B:\left\{B \cdots\left(\mathcal{F}^{\prime}-\beta\right) p\right\} \\
& \text { Mittel } \quad \frac{A+B}{2}-\frac{\mathcal{F}}{2} p+\frac{\alpha+\beta}{2} p \quad ; \quad \frac{\alpha+\beta}{2}=a
\end{aligned}
$$
also

mittl. Ablesung $=A+\frac{\mathcal{F}}{2}-\frac{\mathcal{F}^{\prime}}{2} \frac{\mathcal{F}}{\mathcal{F}^{\prime}+r}+a-a\left(1-\frac{\mathcal{F}}{\mathcal{F}^{\prime}+\boldsymbol{r}}\right)$

$$
=A+a+\frac{\mathcal{F}}{2} \frac{r}{\mathcal{F}^{\prime}+r}-a \frac{\mathcal{F}^{\prime}-\mathcal{F}+r}{\mathcal{F}^{\prime}+r}
$$

Es ist nun gestattet, bei der allgemeinen Kleinheit von $r$ im Vergleich mit $\mathcal{F}^{\prime}$ genähert $z$ u setzen

$$
\frac{\mathcal{F}}{2} \frac{r}{\mathcal{F}^{\prime}+r}=\frac{r \mathcal{F}}{2 \mathcal{F}^{\prime}}\left(1-\frac{r}{\mathcal{F}^{\prime}}\right)=\frac{r}{2} \frac{\mathcal{F}}{\mathcal{F}^{\prime}}\left(1-\frac{r}{\mathcal{F}^{\prime}+r}\right)
$$

folglich :

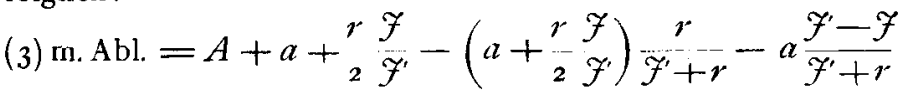
hieraus

Betrachten wir den speciellen Fall: $\mathcal{F}^{\prime}=\mathcal{F}$, so folgt

Prag 1884 Juni. (a) mittl. Abl. $=A+a+\frac{r}{2}-\left(a+\frac{r}{2}\right) \frac{r}{\mathcal{Z}+r}$,

welche Formel in "Albrecht, geogr. Ortsbestimmungen * I. Auflage pag. 49 gegeben erscheint.

Nehmen wir als zweiten Fall: $\mathcal{F}^{\prime}=\frac{\mathcal{Z}}{2}$, welcher häufig bei Universalinstrumenten angetroffen wird, so ergiebt sich als

$$
\text { mittl. Abl. }=A+a+r-(a+r) \frac{2 r}{\mathcal{F}+2 r}+a \frac{\mathcal{F}}{\mathcal{F}+2 r}
$$

und, da mit ausreichender Näherung gesetzt werden kann:

$$
a \frac{\mathcal{F}}{\mathfrak{F}+2 \boldsymbol{r}}=a\left(\mathrm{x}-\frac{2 r}{\mathcal{F}}\right)=a\left(\mathrm{I}-\frac{2 r}{\mathcal{F}+2 r}\right)
$$

so folgt :

(b) $\operatorname{mittl.~Abl.~}=A+2 a+r-(2 a+r) \frac{2 r}{\mathscr{F}+2 r}$

Aehnlich specialisirt sich die obige allgemeine Formel (3) in anderen, besonderen Fällen. Die vierten Glieder der Formeln (a) und (b) werden bei Reduction zahlreicher Ablesungen zweckmässig mit den Argumenten $a+\frac{r}{2}$ resp. $2 a+r$ in Tafeln gebracht.

L. Weinek.

\title{
Ueber die Berichtigung des Schraulenwerthes an Mikrometer-Mikroskopen.
}

Im Anschluss an die Mittheilung von Herrn Prof. Weinek erlaube ich mir eine Bemerkung in Betreff der Berichtigung des »Run der gewöhnlichen Ablese-Mikroskope.

Es macht nicht die geringste Schwierigkeit, das Mikroskop so zu stellen, dass das Bild der Theilung möglichst genau in die Ebene des Mikrometerfadens fällt, und danach durch Messung einiger Intervalle das Verhältniss der Ablesungen $2 u$ der Theilung festzustellen. Wenn man auch die sich ergebenden Correctionen leicht berücksichtigt, so wird es dennoch bei der grossen Menge solcher Ablesungen wünschenswerth sein, diese Correction auf Null oder wenigstens auf ein Mininum zu bringen. Mittelst einer beiläufigen Abmessung der Entfernung des Limbus rom Objective und des Objectivs von der Fadenebene lassen sich nun, wie auch aus Prof. Weinek's Mittheilung hervorgeht, für ein gegebenes Mikroskop oder für einen ganzen Satz gleichartiger Mikroskope, leicht diejenigen Aenderungen der Entfernung des Objectivs und des Mikrometers von dem Limbus genau angeben, welche eine Uebereinstimmung der Trommeltheile mit dem Limbus herbeiführen.

Diese kleinen Aenderungen oder Verstellungen auf das Gerathewohl, so zu sagen, genau auszuführen ist indessen eine müsame zeitraubende Arbeit, die, meiner Erfahrung nach, selten vollständig gelingt. Um unnöthige und unsichere Versuche bei dem Verschieben beider Theile, des Objectivs und des Mikrometers, $2 u$ ersparen, habe ich mich seit längerer Zeit des hier in natürlicher Grösse abgebildeten Hülfsapparates bedient, der für Repsold'sche Mikroskope berechnet war, bei welchen das Objectiv in einem Rohre steckt, welches frei in das äussere Rohr geschoben und dort durch Reibung, resp. Klemmung, festgehalten wird.
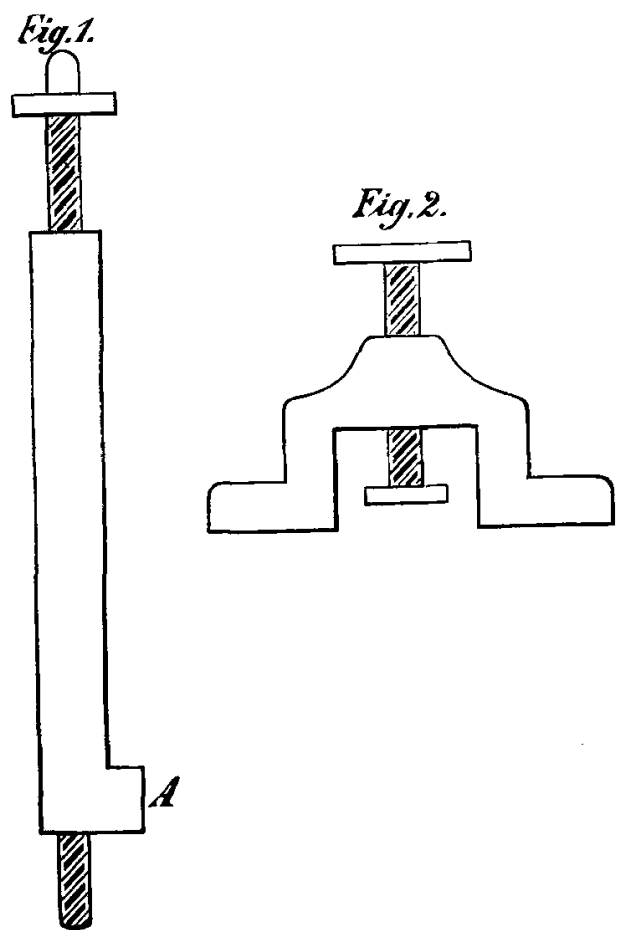
Das Messingstück von quadratischem Querschnitt (Fig. 1) ist der Länge nach durchbohrt und mit einem Schraubengewinde versehen; der Vorsprung bei A ist nach Art eines Zapfenlagers ausgefeilt. Die lange Schraube mit dem Kopfe oben dient nun zur Abmessung der Entfernung des Mikrometers vom Limbus. Ich lasse z. B. den Vorsprung bei A sich gegen das untere Lager des Mikroskopes anlegen und bringe die obere flache Spitze der Schraube mit dem Mikrometerkasten in Berührung. Danach verstelle ich die Schraube um soviel, als die vorhergegangene. Rechnung erfordert, lockere die Lager des Mikroskopes, lasse den Kasten in gleicher Weise berühren und schraube das Mikroskop wieder fest. Man wird ohne Mühe auf ein Hundertstel Millimeter oder noch genauer diese Verschiebung bewerkstelligen und dabei den Faden parallel zu den Limbusstrichen stellen. Zur richtigen Verschiebung des Objectivs dient der in Fig. 2 abgebildete Mikrometerapparat. Das Messingstuck legt sich auf den Rand der Mikroskopröhre; darauf wird mittelst der Schraube die untere kleine Scheibe mit der Objectivfassung in Berührung gebracht. Auch diese Berührung lässt sich sehr genau auffassen: so wie man etwas $\mathrm{zu}$ w geschraubt hat, fühlt man, dass das Messingstück nicht mi gleichzeitig auf beiden Seiten das Mikroskoprohr berül Man stellt danach die Mikrometerschraube in der erford lichen Weise, zieht das Objectivrohr etwas heraus und drü، es wieder soweit zurück, als die Berührung der betreffenc Theile zulässt. Man wird vielleicht diese Correction liel zuerst vornehmen.

In wenigen Minuten ist nun das Mikroskop gen berichtigt, die vorher nothwendige Berechnung der erford lichen Verschiebungen verlangt viel weniger Zeit, da $\mathrm{m}$ für den betreffenden Satz von Mikroskopen ein für alle $\mathrm{N}$ die zu befolgende Regel, im Voraus notirt, zur Ha haben wird.

Je nach der Form der vorhandenen Mikroskope w: man dem Apparat zur Messung der Verstellungen ande vielleicht zweckmässigere, Formen geben. Die Hauptsac bleibt aber immerhin, dass man Versuche auf das Geratl wohl vermeidet.

Kiel 1884 Juni I 8 .

A. Krueger.

\section{Cometen-Beobachtungen}

angestellt mit dem Refractor der k. k. Krakauer Sternwarte.

Comet 1883 i.

\begin{tabular}{|c|c|c|c|c|c|c|c|c|c|}
\hline 1883 & M. Z. Krak. & $\Delta a$ & $\Delta \delta$ & Vergl. & $\alpha$ app. & $\log p \cdot \Delta$ & $\delta$ app. & $\log p \cdot \Delta$ & $*$ \\
\hline Mär 30 & $8^{\mathrm{h}} 23^{\mathrm{m}} 23^{\mathrm{s}}$ & $+1^{m} 65_{5}^{\mathrm{s}}$ & $-13^{\prime} 33^{\prime \prime} 2$ & 7 & $3^{\mathrm{h}} 28^{\mathrm{m}} 3^{6} \cdot 10$ & 9.607 & $+2 I^{\circ} 54^{\prime} 24^{\prime \prime}$ I & 0.774 & $\mathbf{I}$ \\
\hline $3 I$ & $8 \bigcirc 34$ & +048.62 & -228.4 & 8 & $\begin{array}{lll}3 & 33 & 12.05\end{array}$ & $9 \cdot 596$ & $+2 \mathrm{I} 25$ II. 4 & $0.76 \mathrm{I}$ & 2 \\
\hline April $x$ & 81914 & - I 2.84 & +33.9 & 7 & $\begin{array}{lll}3 & 37 & 39.5\end{array}$ & 9.602 & +205551.8 & 0.775 & 3 \\
\hline
\end{tabular}

Mittlere Oerter der Vergleichsterne für I 883.0

\begin{tabular}{|c|c|c|c|}
\hline$\because$ & $\alpha \mathbf{1} 883.0$ & $\delta$ I 883.0 & Autorität \\
\hline I & $3^{\mathrm{h}} 27^{\mathrm{ml}} 29^{\mathrm{s}} 62$ & $+22^{\circ} \quad 7^{\prime} 5^{8.0}$ & BB. VI $+22^{\circ} 500$ \\
\hline 2 & $\begin{array}{lll}3 & 32 & 23.46\end{array}$ & +212740.0 & $W_{2} 3^{\mathrm{h}} 655$ \\
\hline 3 & $33^{8} 4^{2} \cdot 3^{8}$ & $+205^{2} 48.1$ & $W_{2} 3^{\text {h }} 827$ \\
\hline
\end{tabular}

Comet r884 I (Pons r8r2).

\begin{tabular}{|c|c|c|c|c|c|c|c|c|c|c|c|c|}
\hline $1883-84$ & M. Z. Krak. & $\Delta \alpha$ & \multicolumn{2}{|c|}{$\Delta \delta$} & Vergl. & \multicolumn{2}{|r|}{$\alpha$ app. } & $\log p .1$ & \multicolumn{2}{|c|}{$\delta$ app. } & $\log p .4$ & $*$ \\
\hline Oct. 6 & $\mathrm{I} 2^{\mathrm{h}} 34^{\mathrm{m}} \mathrm{O}^{\mathrm{s}}$ & $+0^{\mathrm{m}} 20^{\mathrm{s}} 24$ & $-\mathbf{I}$ & I"57 & 6 & I $6^{\mathrm{h}}$ & $30^{\mathrm{m}} 28.02$ & $9 \cdot 728$ & $+57^{\circ} 43$ & $8 . " 2$ & $0.83 \mathrm{I}$ & I \\
\hline Nov. 20 & IO 1355 & $-0 \quad 2.02$ & -6 & 24.94 & 7 & 17 & 5336 . & & +493 & 31.1 & 37 & 2 \\
\hline $2 \mathrm{I}$ & IO 615 & -o $59.5^{2}$ & -7 & .00 & 6 & I 7 & $5^{6} 5$ & 73 & +4851 & & 40 & 3 \\
\hline 22 & 10 $38 \quad 33$ & to 16.1 6 & +11 & 19.05 & 5 & I 8 & 02 & & $+483^{8}$ & & & 0 \\
\hline 23 & IO 550 & 一 $\quad 56.24$ & +3 & 21.88 & 4 & I 8 & 354 & 10 & +4825 & $5^{2} .8$ & 0.873 & \\
\hline 25 & $\begin{array}{lll}9 & 3^{6} & 37\end{array}$ & +150.52 & -5 & & 8 & I 8 & 1059. & & +4759 & 44 & 04 & \\
\hline 30 & Io $34 \quad 4$ & to 16.17 & $-\mathbf{I} 6$ & & 8 & I 8 & $\begin{array}{ll}31 & 8.45\end{array}$ & 9.635 & +4744 & 46.9 & & 7 \\
\hline Dec. 6 & 10718 & $-039.1 \mathrm{I}$ & +3 & I 7.45 & 7 & I 8 & $5^{8} \quad 44.05$ & 9.656 & $+445 \mathrm{I}$ & 50.3 & $0.84 \mathrm{I}$ & 8 \\
\hline 31 & 74146 & $-03 \mathrm{I} .45$ & 一 I 2 & 50.30 & 6 & $2 \mathrm{I}$ & $3^{8} \quad 43.84$ & 94 & +2415 & 42.9 & $0.73 \mathrm{I}$ & 9 \\
\hline Jan. & $747 \quad 5^{\circ}$ & to 21.09 & -3 & $3^{8.50}$ & 8 & 2 I & $44 \quad 59.72$ & $9.59 \mathrm{I}$ & +2241 & I 5.6 & 0.743 & 10 \\
\hline 2 & $\begin{array}{lll}7 & \text { I I } & 39\end{array}$ & +119.18 & +0 & 24.68 & 8 & 2 I & $\begin{array}{ll}5 \mathrm{I} & 59.5 \mathrm{I}\end{array}$ & 9.554 & $+2 \mathrm{x} \quad 7$ & 32.9 & 0.726 & I I \\
\hline
\end{tabular}

